\title{
四硫富瓦烯三氮唑衍生物的合成及应用研究进展
}

\author{
赵邦屯*, $a$ 陶晶晶 ${ }^{b}$ 陈小纪 $b$ 朱卫民*,b \\ ( ${ }^{a}$ 洛阳师范学院化学化工学院 洛阳 471934) \\ $\left({ }^{b}\right.$ 郑州大学化学与分子工程学院 郑州 450010)
}

\begin{abstract}
摘要 四硫富瓦烯是一类重要的电子供体化合物, 在分子导体和超分子化学领域引起广泛关注. 主要介绍 $\mathrm{Cu}(\mathrm{I})$ 催化的 叠氮和炔基的环加成反应 $(\mathrm{CuAAC}$ )在合成四硫富瓦烯三氮唑衍生物方面的应用进展，主要涉及含端基叠氮的四硫富瓦 烯化合物和含端基炔的反应底物反应以及含端基炔的四硫富瓦烯化合物和含端基叠氮的反应底物反应两类. 同时, 对 利用 CuAAC 点击反应合成的含三氮唑功能基的四硫富瓦烯衍生物在分子识别、分子组装以及分子光电和光伏器件等 光电功能材料领域的应用进展进行综述.
\end{abstract}

关键词＼cjkstart点击反应; 四硫富瓦烯; 三氮唑; 光电功能材料

\section{Progress on Synthesis and Application of Triazole-Based Tetrathiafulvalene Derivatives}

\author{
Zhao, Bangtun $^{*, a} \quad$ Tao, Jingjing ${ }^{b} \quad$ Chen, Xiaoji $^{b} \quad$ Zhu, Weimin ${ }^{*, b}$ \\ ( ${ }^{a}$ College of Chemistry and Chemical Engineering, Luoyang Normal University, Luoyang 471934) \\ ( ${ }^{b}$ College of Chemistry and Molecular Engineering, Zhengzhou University, Zhengzhou 450001)
}

\begin{abstract}
Tetrathiafulvalene (TTF) is an excellent electron donor, therefore it has been used in amyriad of molecular conductors and supramolecular application. The main focus of this review is on the recent progress of synthesis and application of various triazole-based TTF derivatives (TTFs) mainly including traditional tetrathiafulvalene, tetrathiafulvalene vinylogue and extended tetrathiafulvalene via click chemistry. Assisting copper(I)-catalyzed azide-alkyne cycloaddition reaction (CuAAC), the general aspects of TTF molecular design are mainly involved in the reactions of terminal propargyl TTFs with terminal azided substrates as well as the reactions of terminal azided TTFs with terminal propargyl various substrates. This survey is also presented from the view of supramolecular application of the triazole-based TTF systems in molecular recognition, molecular assembly as well as molecular photoelectric and photovoltaic functional materials etc.

Keywords click reaction; triazole; tetrathiafulvalene; photoelectric functional material
\end{abstract}

四硫富瓦烯(Tetrathiafulvalene, 简称 TTF)是一种稳 定可逆的两电子给体, 控制适当的电位, TTF 可以以中 性分子, 自由基阳离子和二价阳离子三种形式存在 ${ }^{[1 \sim 3]}$. 为改善 TTF 的供电子能力和拓展应用研究, 众多 $\pi$ 扩展 TTF 衍生物被设计合成, 其不仅可以导致稳定的氧化态 和聚阳离子体形成, 而且可使分子的 HOMO 和 LUMO 轨道能隙更小, 供电子能力更强 ${ }^{[4,5]}$, 同时通过增加 $\pi \cdots$ $\pi$ 和 $\mathrm{S} \cdots \mathrm{S}$ 作用的数量来提高其维度. 在 $\pi$ 扩展 TTF 衍 生物中, 较常见的是插烯类、苯并类、炔烃类和苯醌类 等, 而基于乙烯基(Tetrathiafulvalene vinylogue, 简称 TTFV)的 TTF 衍生物 ${ }^{[6]}$ 和基于葸醌基扩展的 TTF 衍生物 (extended Tetrathiafulvalene, 简称 exTTF)最为引人关注 (图 1 $)^{[7]}$. 设计合成的四硫富瓦烯类化合物(TTFs)在分子 识别、分子开关, 分子导线、分子梭、传感器、光诱导 或离子诱导电子转移, 以及导电 LB 膜、有机铁磁体、 非线性光学、有机导电和有机光伏材料等研究方面取得 了引人瞩目的成就 ${ }^{[8 \sim 11]}$. 三氮唑基具有芳香性, 可以扩 展分子的共轭区域，也可以起作为桥基来链接功能基 团, 三氮唑衍生物在过渡金属催化、化学生物学和材料 合成等领域发挥了重要作用 ${ }^{[12]}$, 因此, 开展四硫富瓦烯 三氮唑衍生物的合成和应用研究具有重要意义.

\footnotetext{
* Corresponding authors. E-mail: zbt@lynu.edu.cn; zhuwm@zzu.edu.cn

Received February 3, 2017; revised April 7, 2017; published online April 27, 2017.

Project supported by the National Natural Science Foundation of China (No. 21172105).

国家自然科学基金(No. 201172105)资助项目.
} 
<smiles>FC(F)Sc1ccsc1</smiles><smiles>CC(C)c1cccc(C(=C2SC=CS2)C(=C2SC=CS2)c2ccccc2)c1</smiles><smiles>FC(F)(F)C(F)(F)C1=CSC(=c2c3ccccc3c(=C3SC=CS3)c3ccccc23)S1</smiles>

图 1 典型 $\mathrm{TTF}$ 衍生物结构

Figure 1 Typical structures of TTF derivatives

自 Sharpless ${ }^{[13]}$ 和 Meldal $^{[14]}$ 发现点击化学 ${ }^{[15 \sim 19]}$ (Click chemistry) 以来, 由于其反应对氧气、水不敏感, 产物选择性好, 产率较高, 反应处理及产物分离简单方 便的特点, 引起了广泛的研究兴趣. 点击化学反应主要 包括环加成反应、亲核开环反应、非醇醛的羰基化合物 缩合反应和碳碳多键的加成反应等, 其中研究最为广泛 的是 $\mathrm{Cu}(\mathrm{I})$ 催化的端基炔和端基叠氮的 Huisgen ${ }^{[20]} 1,3$-偶 极环加成反应(简称 CuAAC 反应). 利用 CuAAC 反应可 以得到立体专一的 1,4-取代三氮唑衍生物(Eq. 1), 并广 泛应用于生物耦合 ${ }^{[21]}$ 、药物设计 ${ }^{[22]}$ 、高分子合成 ${ }^{[23]}$ 、超 分子 ${ }^{[24]}$ 和材料化学 ${ }^{[25]}$ 等领域.

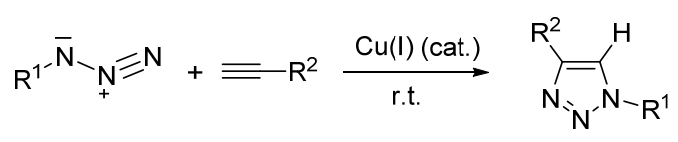

近年来, 由于 $\mathrm{CuAAC}$ 反应的条件普适性强, 利用 点击化学合成含有三氮唑基的四硫富瓦烯衍生物取得 了重要进展, 并成为四硫富瓦烯化学的研究热点之一. 通常 $\mathrm{CuAAC}$ 反应合成 TTFs 衍生物有三类反应: 第一类
是利用含端基炔的 TTF 化合物与含端基叠氮基的底物 反应; 第二类是利用含端基叠氮基的 TTF 化合物与含端 基炔的底物反应; 第三类是利用 $\mathrm{CuAAC}$ 反应, 合成含 有 TTF 单元的超分子功能器件. 本文简要综述利用 CuAAC 反应合成四硫富瓦烯三氮唑衍生物及其功能应 用的研究进展.

\section{1 含端基炔的四硫富瓦烯与对应的含端基叠氮 基的底物反应}

\section{1 基于传统 TTF 的点击反应}

2012 年, Avarvari 等 ${ }^{[26]}$ 利用 CuAAC 和 RuAAC 两种 催化剂将含三甲基硅烷(TMS)保护基的端基炔 TTF 衍生 物 1 和苠基叠氮点击合成 1,4-和 1,5-取代的 1,2,3-三氮唑 TTF 衍生物 2 和 3 (Scheme 1). 循环伏安法实验表明, 异 构体化合物 2 和 3 都是良好的电子供体化合物, 但它们 供电子行为差异较大. 化合物 $\mathbf{2}$ 的第一和第二半波电位 均小于化合物 3 的相应电位, 差值均在 $0.1 \mathrm{~V}$ 附近. 紫外 光谱实验表明, 在分子内存在从 TTF 单元向三氮唑单元 的电子转移过程. 密度泛函理论(DFT)计算结果也反映 了异构体 $\mathbf{2}$ 和 $\mathbf{3}$ 的给电子能力和电子转移行为的差异性. 2014 年, 该课题组利用 RuAAC 反应将双三甲基硅烷 (TMS)保护基的端基炔 TTF 衍生物 4 和芐基叠氮点击合 成 TTF 衍生物 5 和 6 (Scheme 1). 晶体 X 射线分析表明, 晶体 $\mathbf{6}$ 的结构与 $3 \mathrm{~b}$ 的结构差异性大, 主要归因于 $\mathbf{6}$ 中两 个三氮唑基团的空间位阻作用. 紫外可见光谱实验表 明, 化合物 6 存在分子内电荷转移行为 ${ }^{[27]}$, 质子化 $\mathbf{6}$ 的 紫外光谱红移更明显. 为考察功能取代基的影响, 同年,

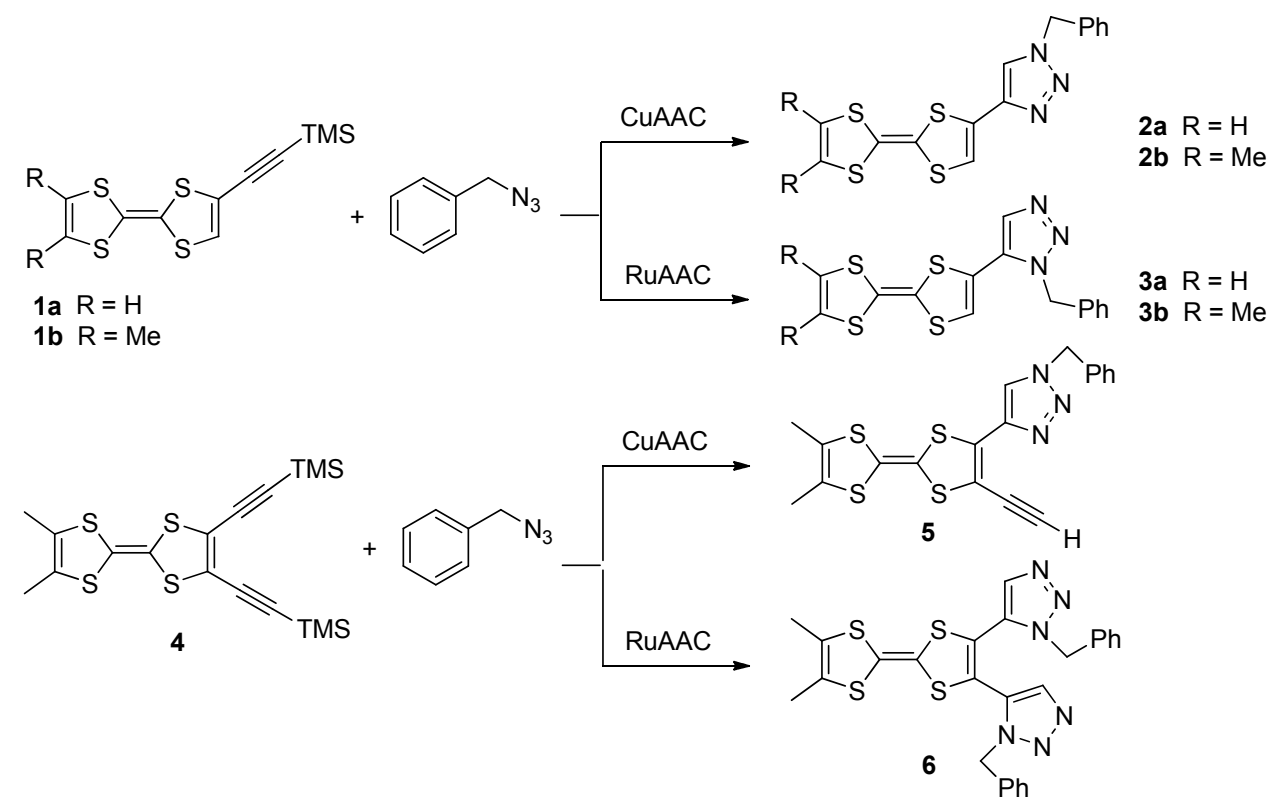

图式 1 端基炔 TTF 和苠基叠氮的反应

Scheme 1 Reactions of terminal alkyne-substituted TTF and benzylazide 
该课题组利用 RuAAC 点击反应合成了含吡啶功能基的 TTF 衍生物 8 和 9 (Scheme 2) ${ }^{[28]}$. 晶体结构表明化合物 9 呈钳子型结构, 两个 TTF 单元平行排列. 循环伏安法实 验表明化合物 9 的第一个氧化峰裂分, 主要是由于分子 中两个 TTF 单元能通过空间发生相互作用, 使得两个 TTF 单元第一氧化电位不同, 说明化合物 9 在溶液中仍 保持钳子型分子结构.

2014 年, Salinas 等 ${ }^{[29]}$ 将含叠氮端基的负载有双吡啶 钓 $\left[\mathrm{Ru}(\text { bipy })_{3}\right]^{2+}$ 染料的硅烷化材料和含端基炔基的 TTF 衍生物 10 和 11 利用 $\mathrm{CuAAC}$ 反应制备出新型的门控介 孔材料 12 和 13 (Scheme 3), 利用 TTF 的供电子性质, 其 可以与含硝基芳香类炸药如三硝基苯甲硝胺(Tetryl)、三 硝基甲苯(TNT)、三硝基苯(TNB)形成电荷转移复合物. 实验表明此门控介孔材料可以作为硝基芳香类炸药的 荧光检测探针而广泛应用。

\section{2 基于 TTFV 的点击反应}

开发基于 TTF 的苂光传感器是超分子化学中的一
个热门课题 ${ }^{[30,31]} .2012$ 年， Zhao 等 ${ }^{[32]}$ 利用 $\mathrm{CuI}$ 催化剂将 含端基炔的乙烯基 TTFV 衍生物 14 和 9-叠氮甲基葱反 应，点击合成新型 TTFV 衍生物 15 (Eq. 2), 其中央 TTFV 核心作为电子供体，两个葱基团作为苂光单元， 两个三氮唑环既为电子供体和荧光单元的连接基, 又为 键合金属离子的受体单元. 苂光和紫外光谱实验表明, TTFV 15 可作为 $\mathrm{Cu}^{2+} 、 \mathrm{Fe}^{2+}$ 和 $\mathrm{Cd}^{2+}$ 光谱探针, 是首例基 于 TTFV 的光谱离子传感器, 其检测限达到微摩尔浓度 级水平, 有望在环境修复、生物学研究和工业分析等领 域广泛应用. 2013 年, 该课题组利用同样的方法合成了 基于 TTFV 的含稠环芳烃和大环的 TTF 系列衍生物 17 21 (Scheme 4), 并通过紫外和苂光光谱详细研究了 它们分别与富勒烯 $\mathrm{C}_{60}$ 和 $\mathrm{C}_{70}$ 的超分子相互作用行为, 结 果表明分子钳式的 TTFV 衍生物 $\mathbf{1 7}$ 和大环结构 $\mathbf{2 0}$ 与富 勒烯作用很弱, 而化合物 18, 19 和 21 与富勒烯作用较 强, 并可以用来分离富勒烯 $\mathrm{C}_{60}$ 和 $\mathrm{C}_{70}{ }^{[33]}$.

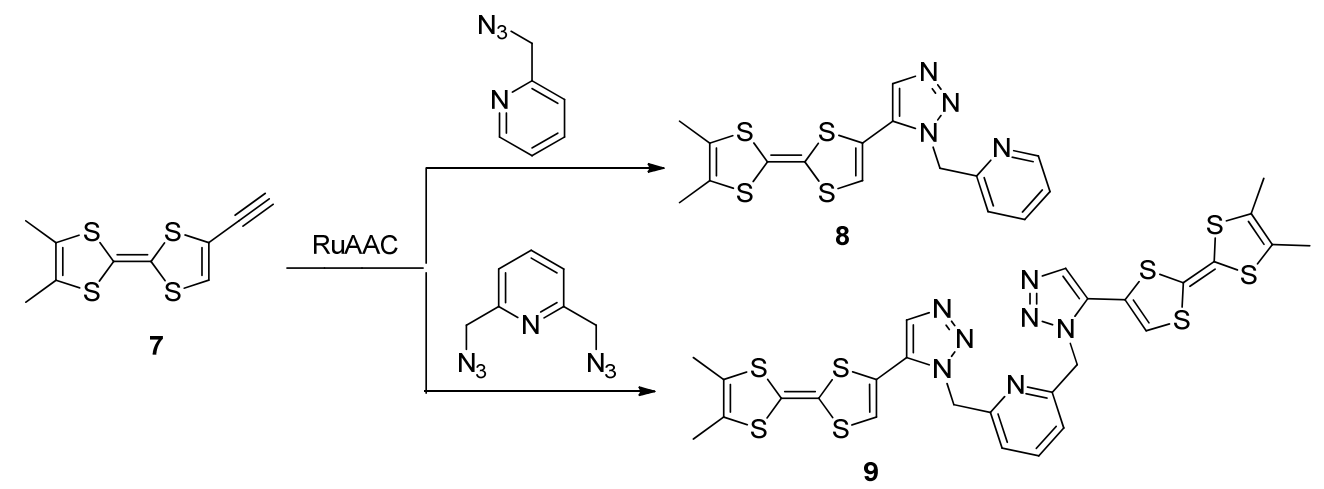

图式 2 含吡啶基的 TTF 三氮唑衍生物的合成

Scheme 2 Synthesis of triazole-based TTF derivatives with pyridine group
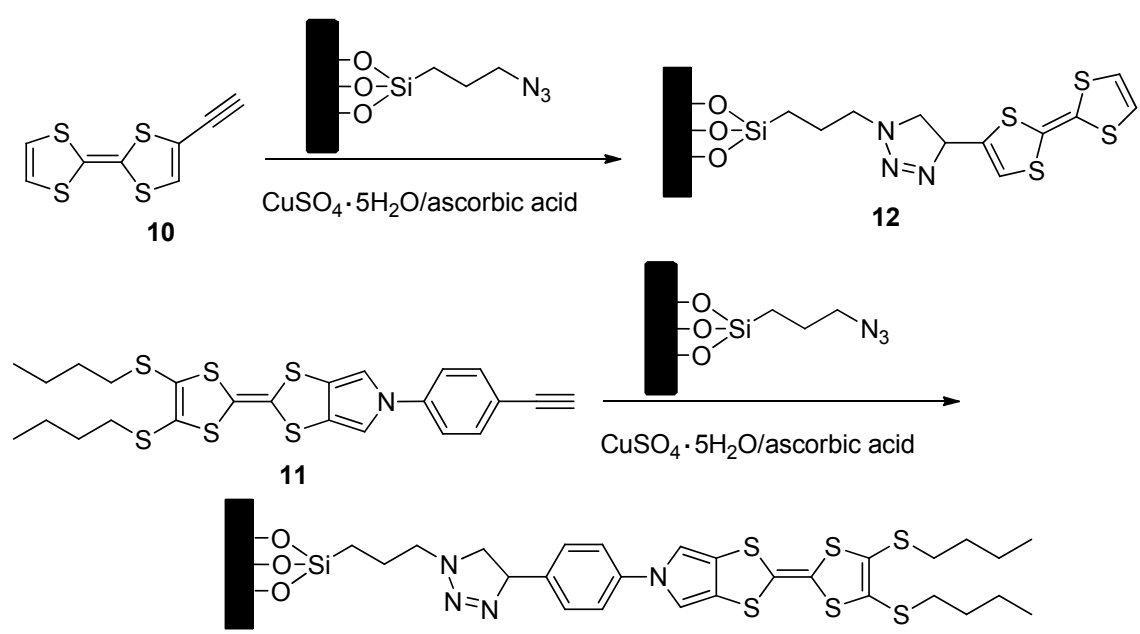

13

图式 $3 \mathrm{CuAAC}$ 反应制备新型 TTF 衍生物的硅门控介孔材料

Scheme 3 Preparation of silica mesoporous supports gated with TTF derivatives by CuAAC reaction 

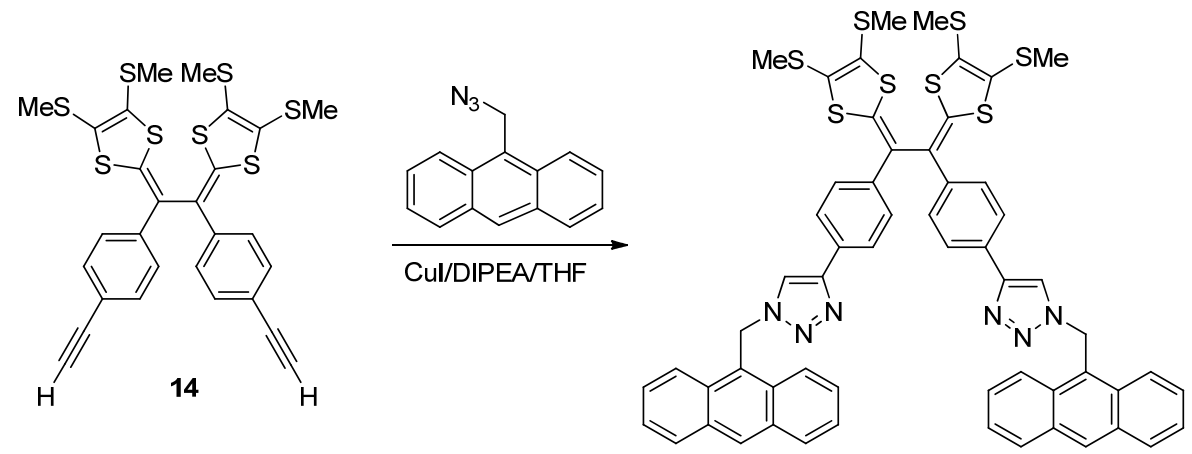

(2)

15
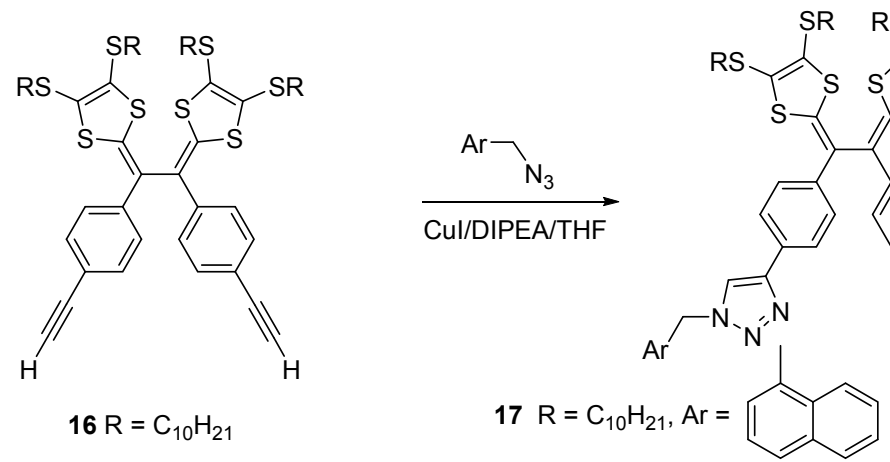<smiles>[R]C1=C(S)SC(=Cc2ccc(-c3cn(C[Al])nn3)cc2)S1</smiles>

$$
16 \mathrm{R}=\mathrm{C}_{10} \mathrm{H}_{21}
$$

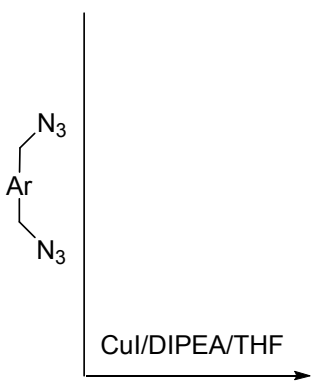

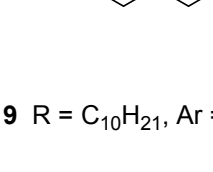

$18 \mathrm{R}=\mathrm{C}_{10} \mathrm{H}_{21}, \mathrm{Ar}=$<smiles>Cc1c2ccccc2cc2ccccc12</smiles><smiles>Cc1ccc2ccc3cccc4ccc1c2c34</smiles>

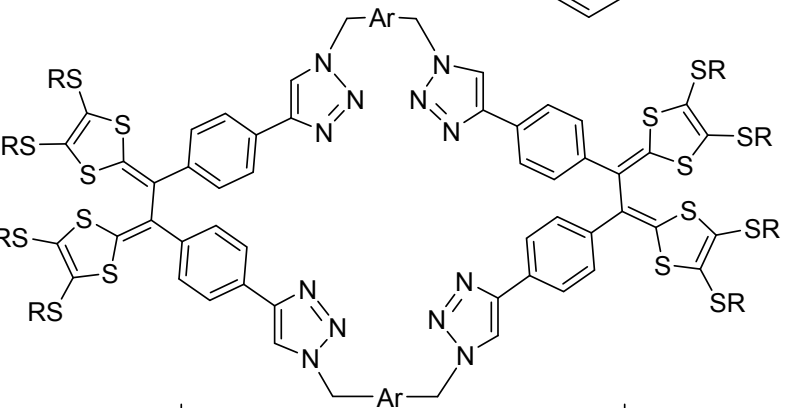

$20 \mathrm{R}=\mathrm{C}_{10} \mathrm{H}_{21}, \mathrm{Ar}=$<smiles>Cc1ccc(C)cc1</smiles>

$21 \mathrm{R}=\mathrm{C}_{10} \mathrm{H}_{21}, \mathrm{Ar}=$<smiles>Cc1c2ccccc2c(C)c2ccccc12</smiles>

图式 4 含三氮唑基的 TTFV 的合成

Scheme 4 Synthesis of triazole-based TTFV derivatives

生物传感器一直是生物分子检测器研究热点之 - ${ }^{[34]}$. 糖类分子传感器通常包含糖分子受体和信号输 出以及二者之间的链接基三个单元. 苯硼酸单元作为糖 类高效分子受体可以在水相中与二元醇、多元醇等形成 苯硼酸酯. 信号输出单元通常是利用有机发色团、荧光 基团和电化学活性基团等光电活性基团. 链接基通常要 满足识别和信号表达效能以及合成可能性等要求. 因 此, 基于苯嗍酸基的糖类分子传感器有比色 ${ }^{[35]}$ 、苂光 ${ }^{[36]}$ 和电化学 ${ }^{[37]}$ 等各种类型.
2014 年, Zhao 等 ${ }^{[38]}$ 将含端基炔的乙烯基 TTFV 衍生 物 14 和邻叠氮甲基苯硼酸酯利用 $\mathrm{CuI}$ 催化剂, 点击合成 TTFV 衍生物 22, 其经水解得到新型基于 TTFV 的硼酸 化合物 23 (Scheme 5). 电化学(CV 和 DPV) 实验表明, 在 磷酸盐缓冲 $(\mathrm{pH}=7.41)$ 的 $\mathrm{DMSO}-\mathrm{H}_{2} \mathrm{O}$ 溶液中, 化合物 $\mathbf{2 3}$ 可以和不同单糖显示选择性电化学反应，可作为潜在应 用的糖类传感器. 同时化合物 22 可以选择性电化学识 别 $\mathrm{F}^{-}$离子. 


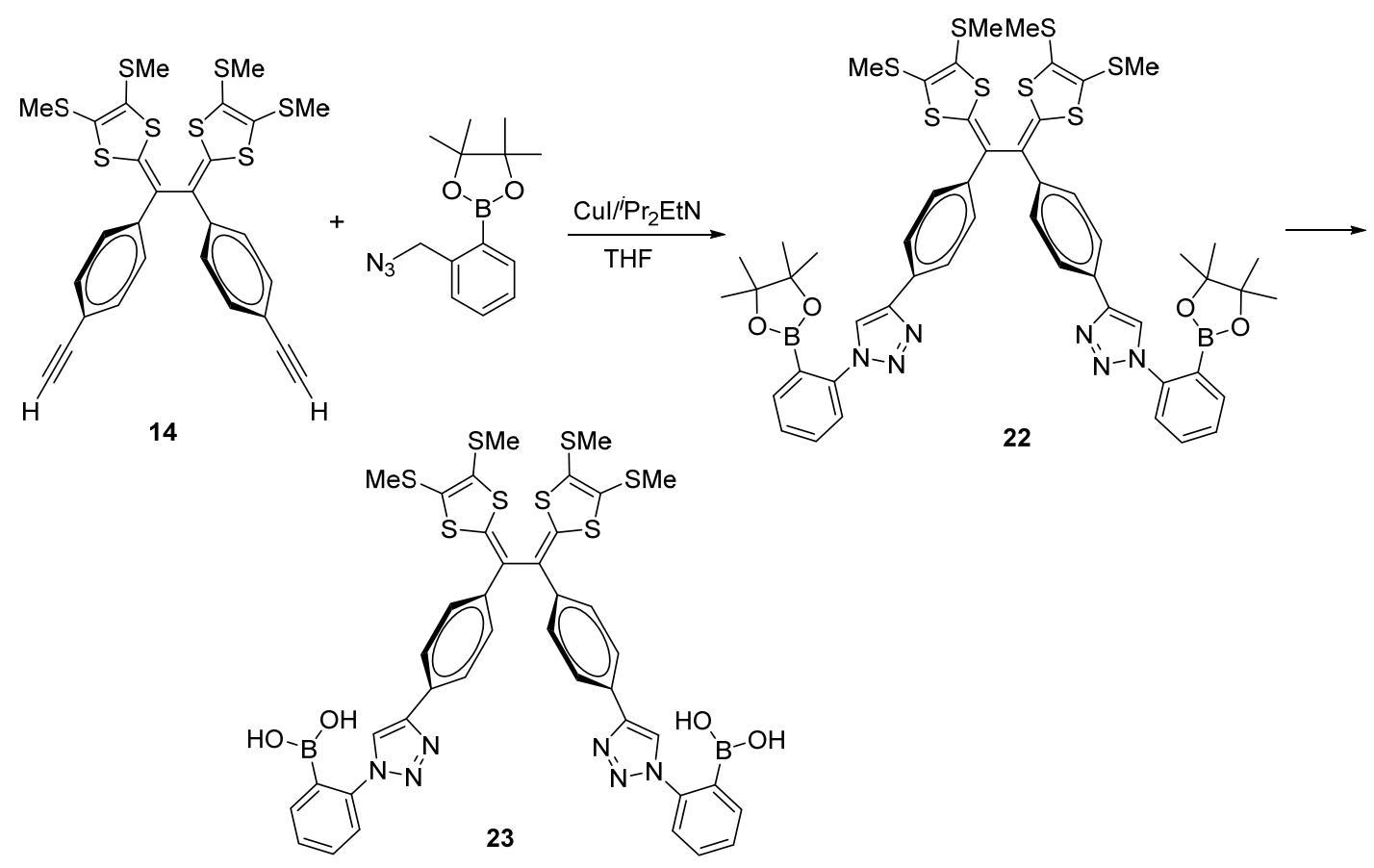

图式 5 含苯嗍酸基的 TTFV 衍生物的合成

Scheme 5 Synthesis of TTFV derivative with phenylboronic groups

\section{3 基于 exTTF 的点击反应}

2010 年, Zhao 等 ${ }^{[39]}$ 将含双端基炔的蒽醌基 exTTF 衍生物 24 和邻叠氮甲基苯硼酸酯利用 $\mathrm{CuI}$ 催化剂, 点击 合成 exTTFV 衍生物 25 (Scheme 6). 电化学(DPV) 实验 表明, 在磷酸盐缓冲 $(\mathrm{pH}=8.75)$ 的 $\mathrm{DMSO}-\mathrm{H}_{2} \mathrm{O}$ 溶液中, 化合物 25 可以与果糖和核糖选择性电化学识别反应, 这是首例基于 exTTF 的糖类分子传感器. 由于化合物 25 在水溶液中的溶解度和溶液粘度等限制了其定量应 用范围，因此，修饰开发新型的基于 TTF 的糖类分子传 感器是一个重要方向.

\section{2 含端基叠氮基的四硫富瓦烯与对应的含端基 炔的底物反应}

\section{1 基于传统 TTF 的点击反应}

芢二酰亚胺(PDI)由于其高荧光量子产率和光热化 学耐持久性可以作为有机太阳能电池和人工光合作用 领域的研究与开发. 2009 年, Nielsen 等 ${ }^{[40]}$ 以 $\mathrm{CuSO}_{4}$ • $5 \mathrm{H}_{2} \mathrm{O}$-抗坏血酸为催化剂, 将端基叠氮化 TTF 衍生物 26 与端基炔的 PDI 衍生物反应, 点击合成含有 TTF 电子给 体和 PDI 电子接受体的二联体化合物 27 (Eq. 3). 原子力 显微(AFM) 实验表明, 化合物 TTF-PDI 二联体 27 在云母 表面组装形成纳米线.

基于氧化还原开关的 TTF 离子传感器研究已经受 到广泛关注. 喹啉化合物能够键合金属离子, 并且自身 具有荧光性质, 将其与 TTF 单元结合, 有望开发具有光<smiles>CC#Cc1ccc2c(=C3SC(S(C)(=O)=O)=C(S(C)(=O)=O)S3)c3cc(C#CC)ccc3c(=C3SC(S(C)(=O)=O)=C(S(C)(=O)=O)S3)c2c1</smiles>

24<smiles>CSC1=C(S(C)(=O)=O)SC(=c2c3cc(-c4cn(Cc5ccccc5B(O)O)nn4)ccc3c(=C3SC(S(C)(=O)=O)=C(S(C)(=O)=O)S3)c3cc(-c4cn(Cc5ccccc5B(O)O)nn4)ccc23)S1</smiles>

图式 6 含苯嗍酸基的 exTTF 衍生物的合成

Scheme 6 Synthesis of exTTF derivative with phenylboronic groups

电信号检测的离子传感器. 2012 年, Zhao 等 ${ }^{[41]}$ 利用 $\mathrm{CuI}$ 催化剂, 将含端基叠氮的 TTF 衍生物 28 与 8 -炔丙基取 代的喹啉化合物反应，点击合成了含三氮唑基和喹啉基 的 TTF 衍生物 29 (Eq. 4). 循环伏安法实验表明, 化合物 29 对 $\mathrm{Zn}^{2+}$ 离子有一定的响应能力. 苂光滴定光谱和核 

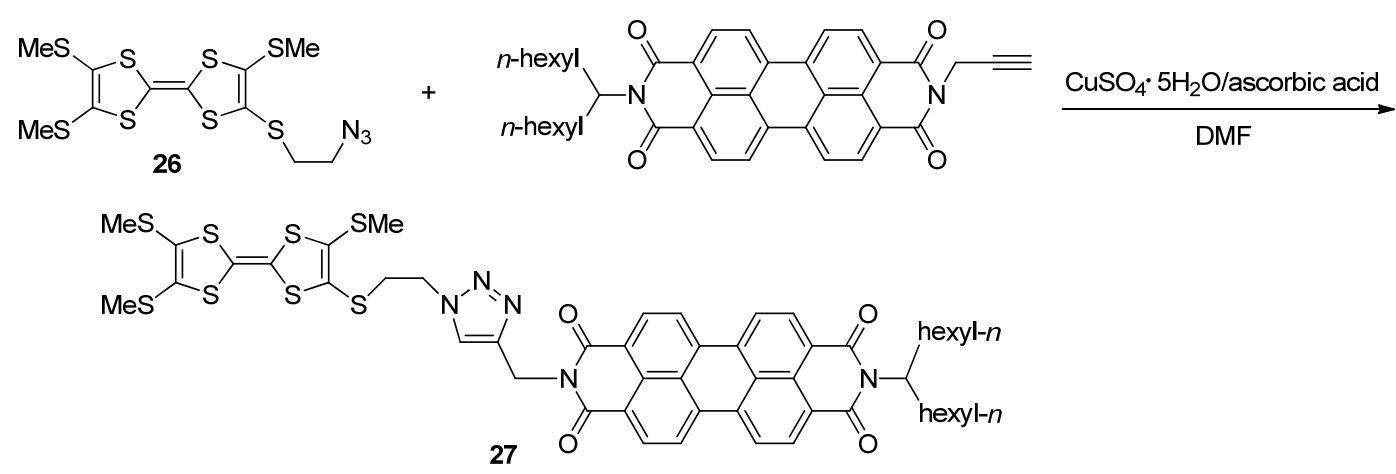

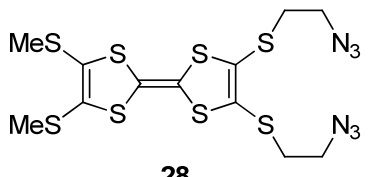

28

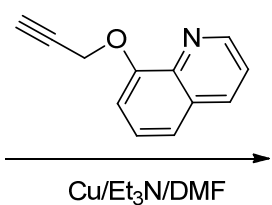

$\mathrm{Cu} / \mathrm{Et}_{3} \mathrm{~N} / \mathrm{DMF}$

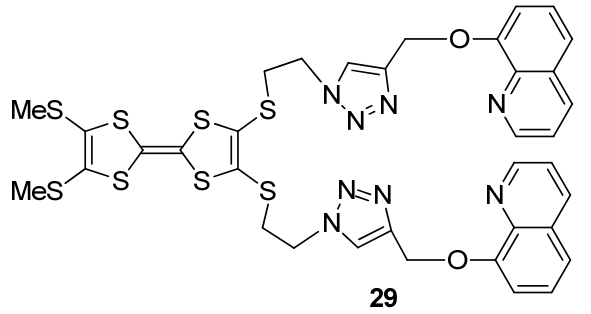

29

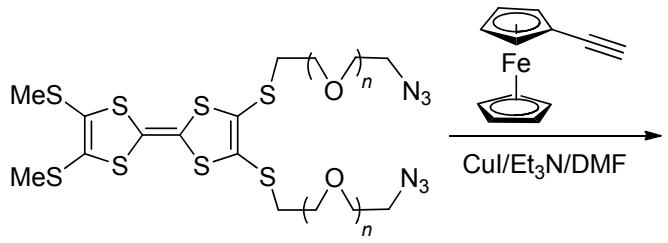

$28 n=0$

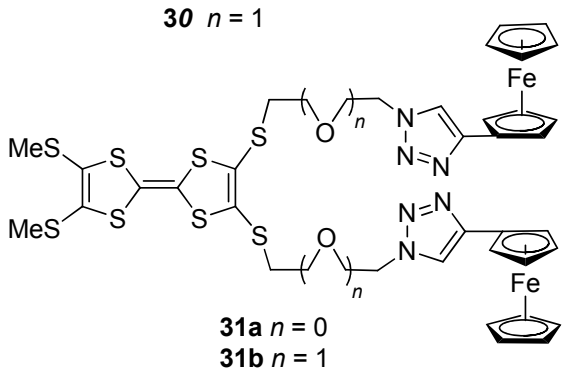

$31 \mathrm{~b} n=1$

饰葱醌反应，点击合成了 1,8 -二羟基葱醌-四硫富瓦烯 衍生物 33 (Eq. 6). 苂光滴定光谱实验表明化合物 32 对 $\mathrm{Al}^{3+}$ 离子有很好的识别能力, 有望作为 $\mathrm{Al}^{3+}$ 离子的苂光 传感器. 紫外滴定光谱实验表明, $\mathrm{Sc}^{3+} 、 \mathrm{Al}^{3+}$ 和 $\mathrm{Pb}^{2+}$ 离子 能够有效促进化合物 33 和四氯对苯醌之间的分子间电 子转移.

杯芳烃作为第三代超分子主体化合物得到了广泛 的研究和应用, 其中 CuAAC 点击反应在杯芳烃衍生的 合成及其分子识别方面获得了广泛的关注 ${ }^{[44]} .2013$ 年, Zhao 等 ${ }^{[45]}$ 首次利用 $\mathrm{CuSO}_{4} \cdot 5 \mathrm{H}_{2} \mathrm{O}$-抗坏血酸钠 $(\mathrm{VcNa})$ 催 化剂, 将含叠氮端基 TTF 衍生物 $\mathbf{2 6}$ 和 $\mathbf{2 8}$ 分别与下缘含 端基炔的杯芳烃发生点击反应得到三氮唑基连接的杯 芳烃-四硫富瓦烯二联体 34 和 35 (Scheme 7). 循环伏安 法实验表明, 34 和 35 均显示可逆两电子氧化还原行为. 同时, 紫外光谱实验研究了金属离子如 $\mathrm{Pb}^{2+} 、 \mathrm{Sc}^{3+}$ 、 

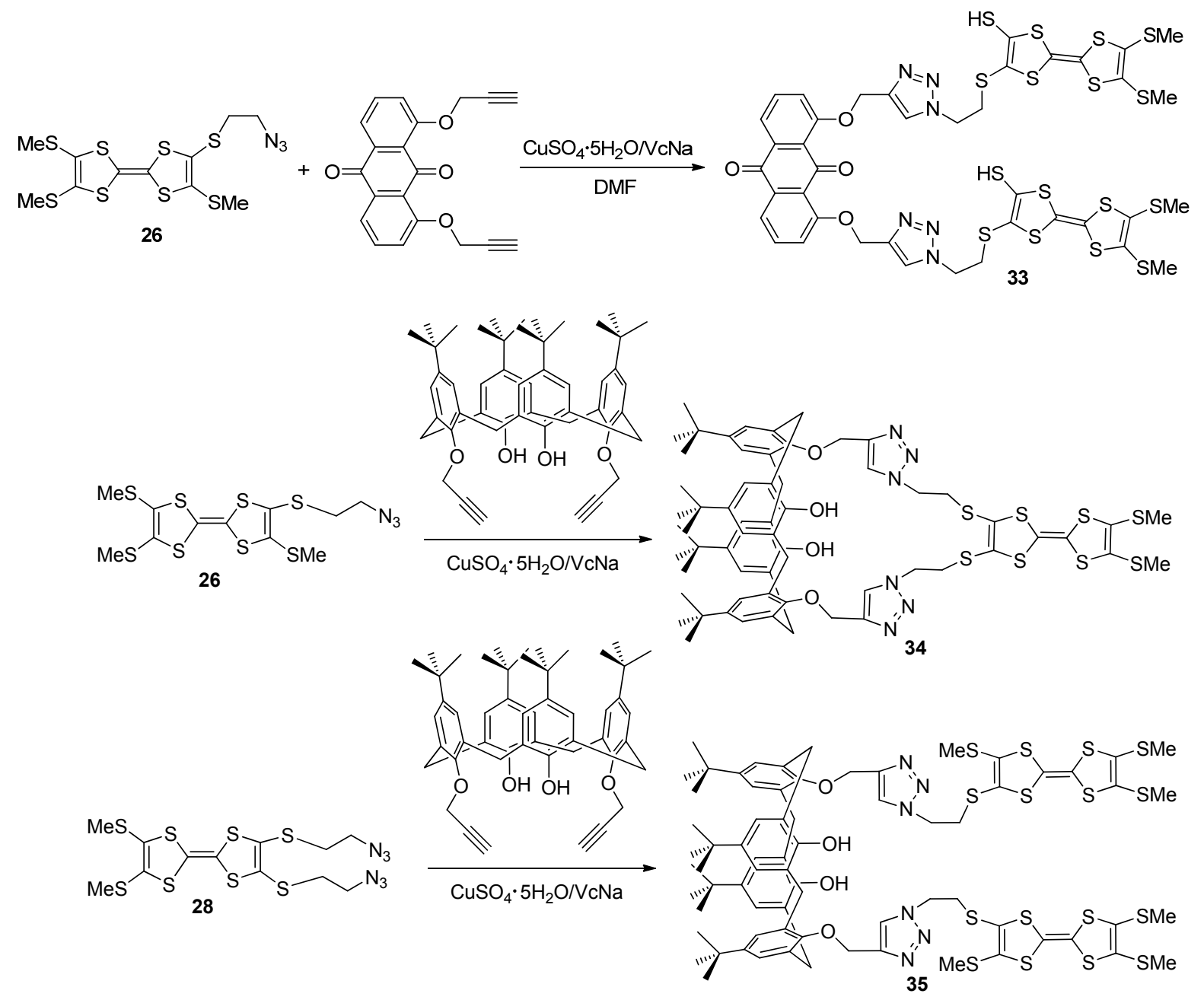

图式 7 三氮唑基连接的杯[4]芳烃-四硫富瓦烯二联体的合成

Scheme 7 Synthesis of the triazole-linked calix[4]arene-tetrathiafulvalene dyads

$\mathrm{Zn}^{2+}$ 诱导的 34 和 35 分别与四氯对苯醌分子间的电子转 移行为, 结果表明不同的金属离子对电子转移的影响不 同. 对于同一金属离子, 化合物 $\mathbf{3 5}$ 的电子转移能力大于 34, 表明配位作用、构象和分子柔韧性等对分子间电子 转移行为有重要影响, 这对于开发基于 TTF 超分子的分 子间电子转移体系具有重要意义.

2015 年, $\mathrm{Zhao}$ 等 ${ }^{[46]}$ 利用 $\mathrm{CuSO}_{4} \cdot 5 \mathrm{H}_{2} \mathrm{O}$-抗坏血酸钠 ( $\mathrm{VcNa}$ )催化剂, 通过 $\mathrm{CuAAC}$ 点击反应将含叠氮端基的 TTF 衍生物(26、28、36、37)和含端基炔的硫桥杯芳烃 (TCA) 合成得到 TTF-TCA 二联体化合物 38 41 (Scheme 8). 通过紫外光谱实验探讨在非氧化性金属离子存在 下, TTF-TCA 二联体 38、39、40 和 41 分别与四氯对苯 醌的分子间电子转移行为, 结果表明 $\mathrm{Sc}^{3+}$ 促进电子转移 行为最为有效. 在同一金属离子存在下, 化合物 41 和 40 比化合物 39 和 38 表现出更高效的电子转移行为.

\section{3 基于 TTF 点击反应构筑分子器件}

随着超分子化学的发展, 具有特殊拓扑结构的分子
构造体如索烃、轮烷、准轮烷等引起了化学家浓厚兴趣. 通过以分子构造体为基本元件研制的分子内存芯片、分 子阀等分子器件, 使得在超分子层次上完成信息和能量 的转换、传输、存储等功能变为现实 ${ }^{[47]}$. 拥有 “箱型” 空穴和刚性的缺 $\pi$ 电子联吡啶基团的对二甲苯基 4,4-联 吡啶环蕃 $(\mathrm{CBPQT})$ 可以与富 $\pi$ 电子芳香类化合物形成电 子转移复合物. 近年来, 以环蕃 CBPQT 为模板来构筑 分子轮烷已经取得重要成果, 尤其是与富 $\pi$ 电子 $\mathrm{TTF}$ 体 系的分子器件研究最为关注 ${ }^{[48 \sim 52]}$.

2007 年, Stoddart 等 ${ }^{[53]}$ 基于 “先穿后封” 的模板导 向合成策略，利用含端基叠氮并拥有䒺基的 TTF 衍生物 42 作为电子供体与对二甲苯基桥联的联吡啶环番 (CBPQT)电子接受体形成电子授受体准轮烷, 然后再与 含端基炔封端基底物发生 $\mathrm{CuAAC}$ 点击反应，合成构筑 分子轮烷 43 (Eq. 7). 循环伏安法和紫外光谱实验表明, 中性状态轮烷 43 中 TTF 与 CBPQT 单元之间存在分子 内电荷转移, 而当 $\mathrm{TTF}$ 被氧化处于二价阳离子状态时, CBPQT 与菜单元之间形成电子授受体, 实验表明 


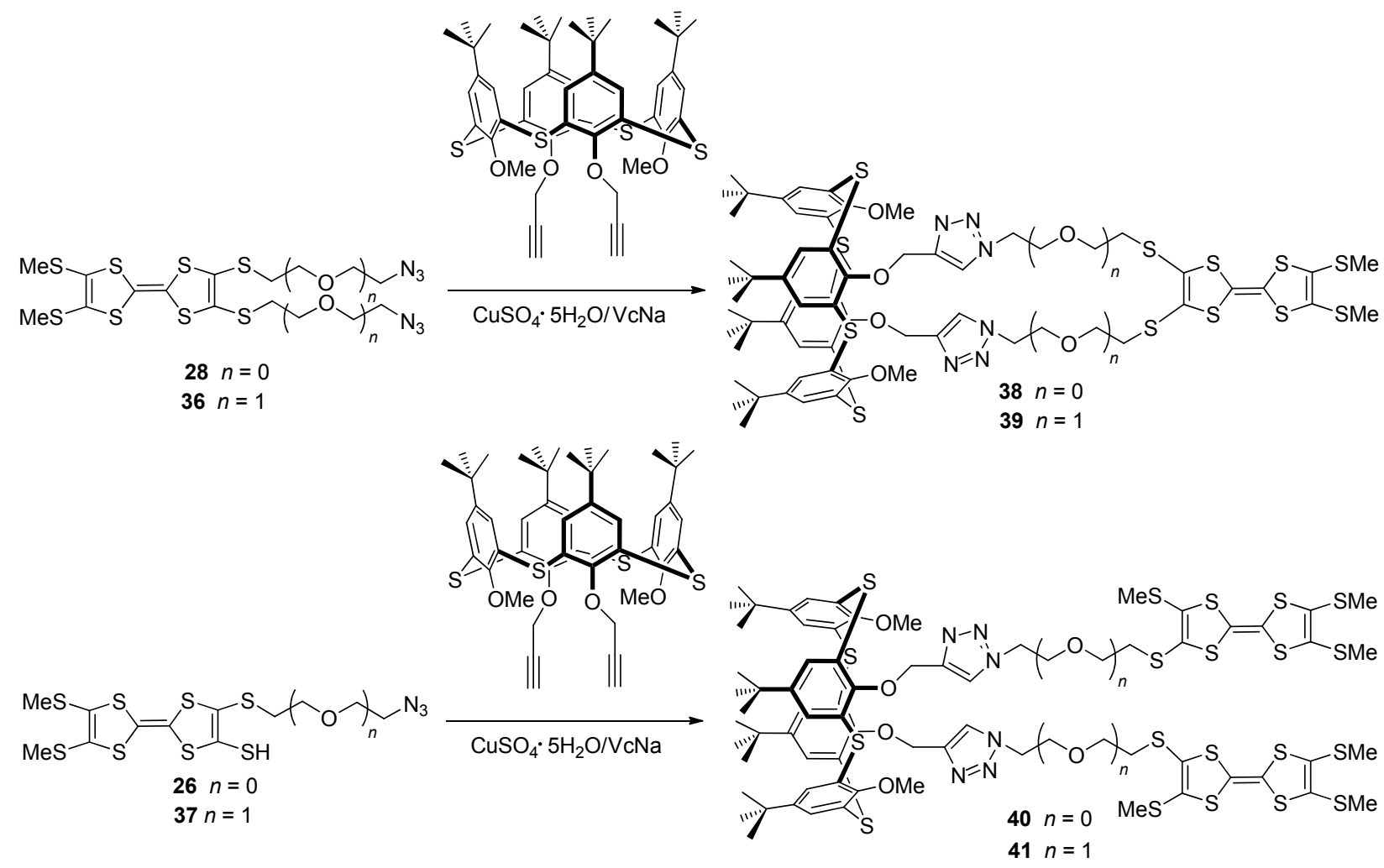

图式 8 三氮唑基连接的硫桥杯 [4]芳烃-四硫富瓦烯二联体的合成

Scheme 8 Synthesis of the triazole-linked thiacalix[4]arene-tetrathiafulvalene dyads
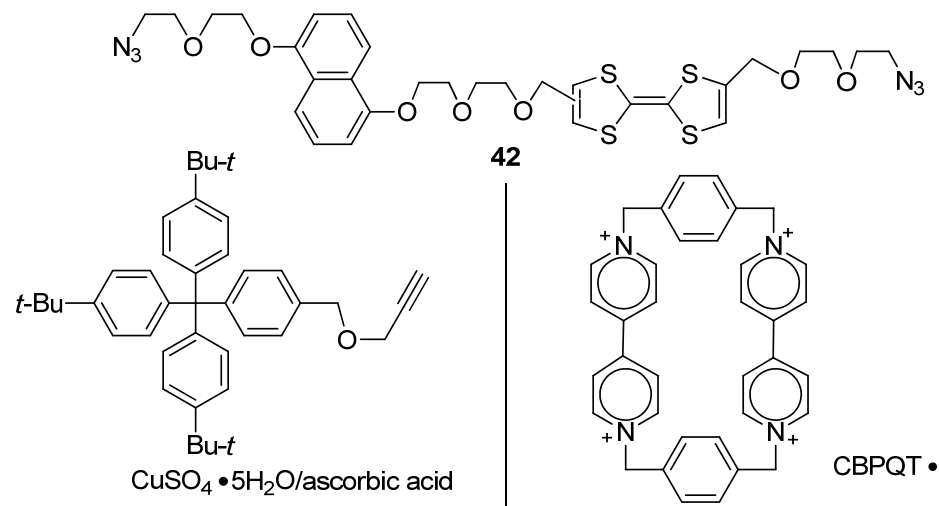

42

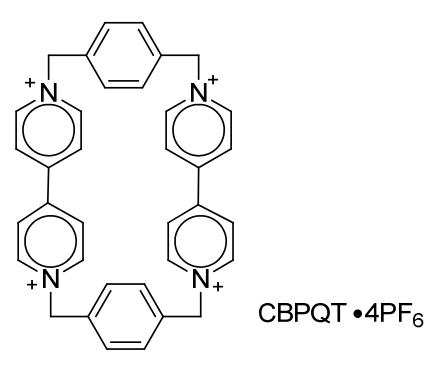

$\downarrow$
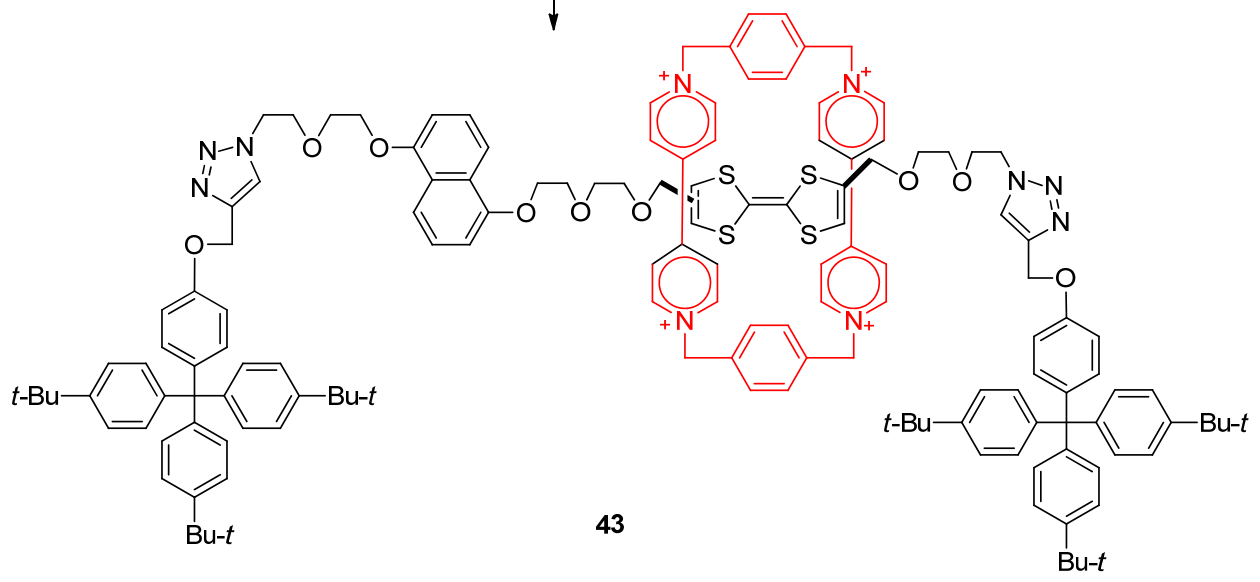
行为. 同年, Stoddart 等 ${ }^{[54]}$ 基于该原理, 利用 $\mathrm{TTF}$ 衍生物 42 和拥有醚链连接疏水基的端基炔底物发生 CuAAC 点 击反应, 得到双稳态分子轮烷 44 (Eq. 8). 轮烷 44 中轮 烷和两端介晶封端基共同促使构筑具有层状液晶状态, 进而组装成更加复杂并且在较宽的温度范围内(室温〜 $150{ }^{\circ} \mathrm{C}$ ) 可以保持的碟状近晶相, 光谱电化学实验表明, 双稳态分子轮烷 44 可作为电化学调控的液晶材料.

2008 年, Stoddart 等 ${ }^{[55}$ 基于模板导向合成策略, 利 用含叠氮端基的三角型 $\mathrm{TTF}$ 衍生物 45 与电子受体 CBPQT 形成准轮烷, 然后与含端基炔的封端基底物发 生 $\mathrm{CuAAC}$ 点击反应制得双稳定的三脚架型分子轮烷 46 (Eq. 9). 光谱电化学实验表明, 与类似的双稳定分子轮 烷不同, 三脚架型分子轮烷 46 中稳定存在 TTF 自由基 阳离子二聚体, 对于开发基于 TTF 的分子轮烷具有重要 指导意义. 同年, Stoddart 等 ${ }^{[56]}$ 将一端含醚链连接封端 基，另一端含醚链连接叠氮端基的 $\mathrm{TTF}$ 衍生物 47 与 $\alpha$ 环糊精混合, 然后利用 $\mathrm{CuAAC}$ 点击反应与含端基炔的 封端基底物反应, 得到了基于 $\alpha$ 环糊精的 TTF 氧化还原 型轮烷 48 (Eq. 10). 诱导圆二色谱(ICD)实验和 ${ }^{1} \mathrm{H}$ NOESY 实验表明, 轮烷 $\mathbf{4 8}$ 中 TTF 单元被 $\alpha$ 环糊精的疏水
空腔包裹. 紫外光谱实验也表明轮烷 $\mathbf{4 8}$ 中 TTF 的吸收 峰由于 $\alpha$ 环糊精的包裹而发生吸收强度下降且显著红 移. 循环伏安实验表明由于包裹作用 TTF 的第一氧化电 位明显增大，而第二氧化电位几乎不变，表明控制电位 $\alpha$ 环糊精可以在轮烷中移动. ${ }^{1} \mathrm{H}$ NOESY 实验进一步表 明电化学可以调控供 $\alpha$ 环糊精在 TTF 单元和三氮唑单元 之间移动, 这对于构筑电化学调控的纳米药物传输分子 体系和器件具有重要指导作用. 分子动态模拟(MD)计 算表明，基于环糊精的带电荷分子轮烷中分子梭运动的 驱动力是水分子与轮烷组成部分的作用 ${ }^{[57]}$.

2011 年, Stoddart 等 ${ }^{[58]}$ 设计构筑了更为复杂的含 TTF 单元和对二甲联苯基桥联的联吡啶环番的机械互 联锁结构 49(图 2). 实验表明 49 诱导氧化后, 存在平移 和旋转运动, 该研究对实现完成从简单分子开关的转变 到多态变换的复杂分子机器构筑以及提高分子器件的 信息存储具有重大意义.

石墨烯具有二维碳六角晶格结构，其显著的大比表 面积和电、热、机械性能引起了在新型复合材料、生物 传感和催化剂领域的研究兴趣. 2012 年, Szunerits 等 ${ }^{[59]}$ 首先将含叠氮端基 $\mathrm{TTF}$ 衍生物 $\mathbf{5 0}$ 和氧化石墨烯(GO)超

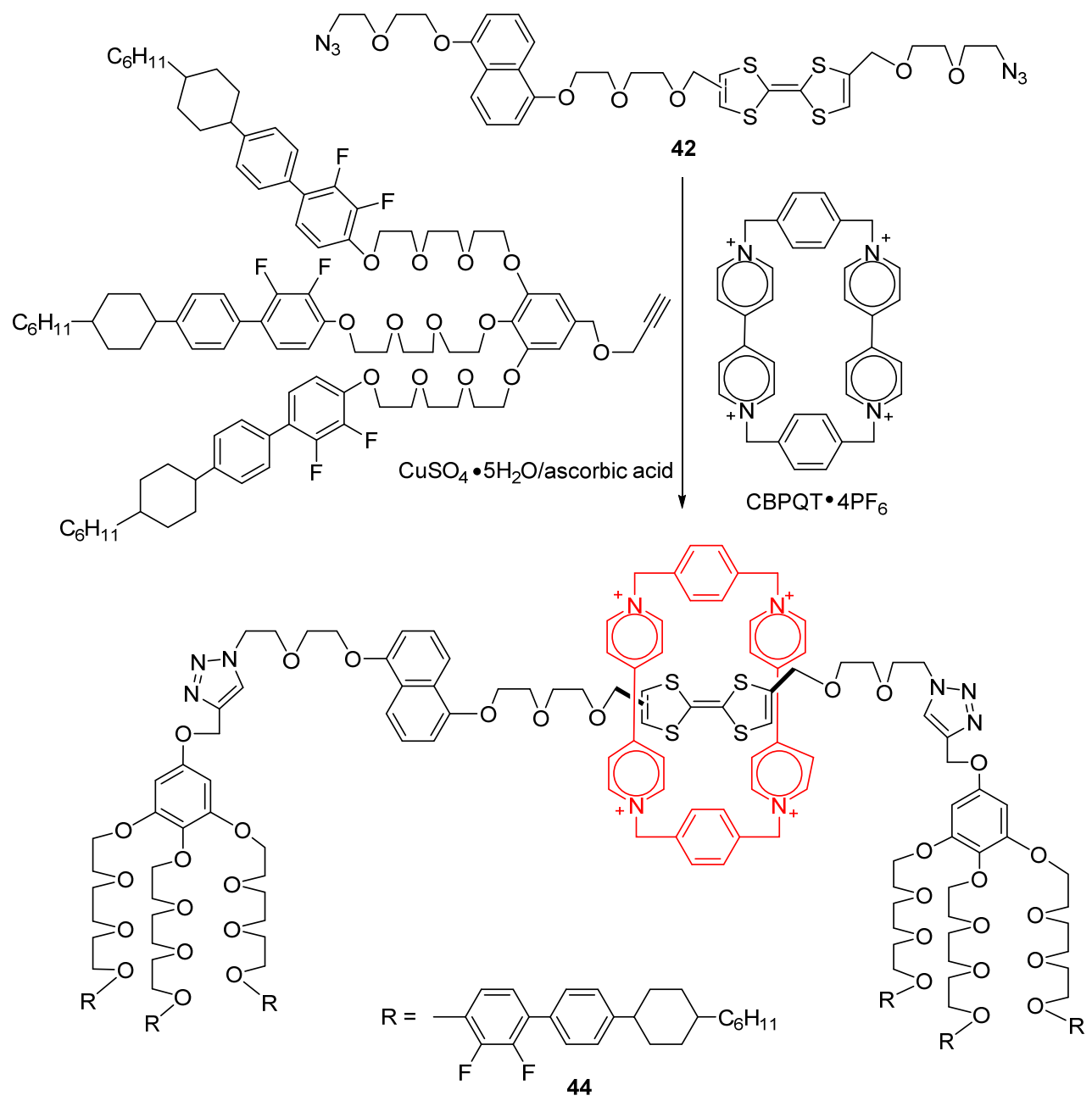



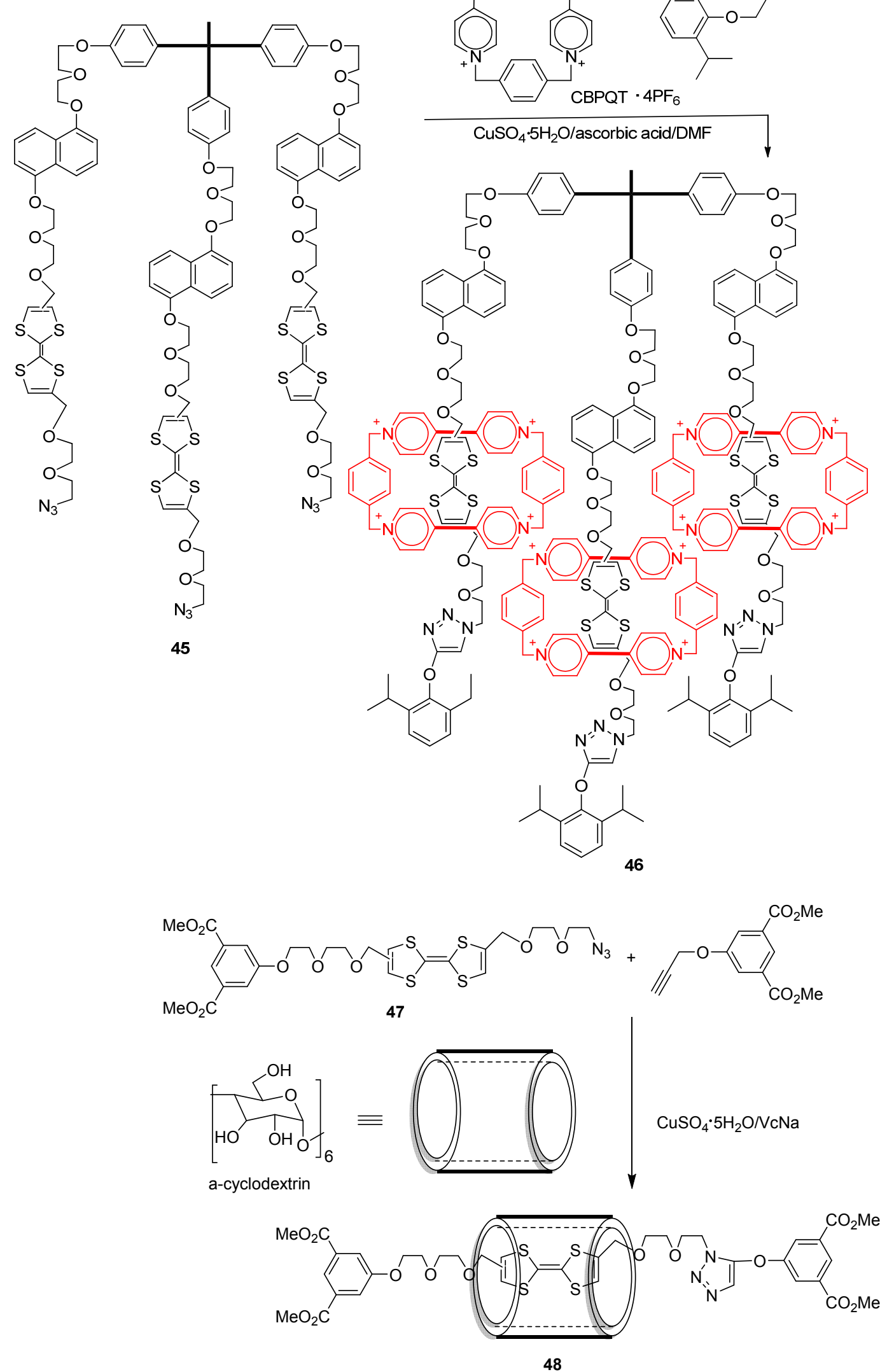


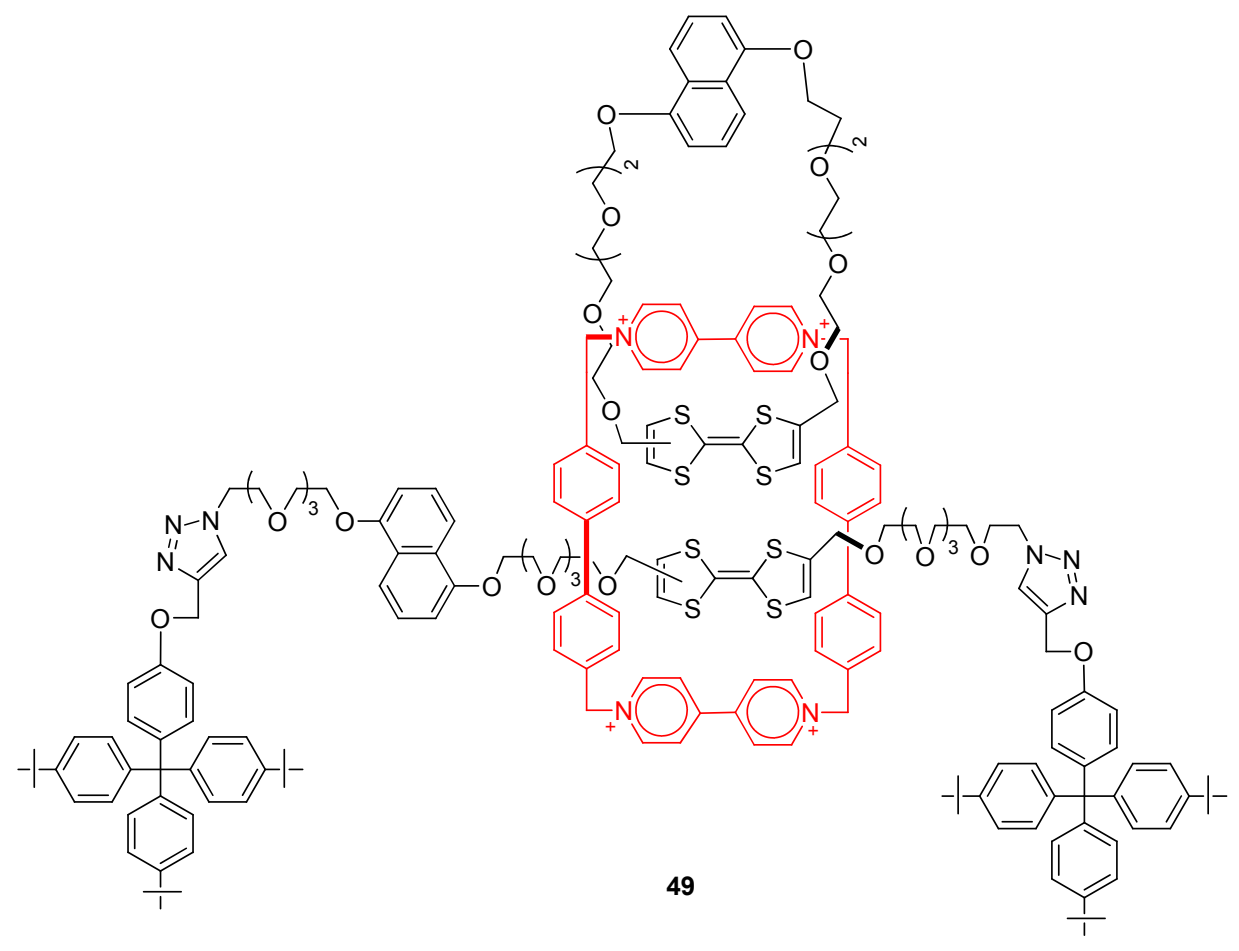

图 2 基于 $\mathrm{TTF}$ 三氮唑的[3]索烃

Figure 2 Switchable[3]rotacatenane with triazole-based TTF derivative

声溶解, 利用 TTF 的供电子性质将氧化石墨烯(GO)还 原来构筑基于石墨烯的纳米杂化材料. 然后利用 $\mathrm{CuAAC}$ 点击反应将其作为基质与含端基炔的甘露糖反
应得到含糖的石墨烯材料(Scheme 9). 通过 $\mathrm{Fe}\left(\mathrm{ClO}_{4}\right)_{3}$ 氧 化作用可以控制糖从该石墨烯基质材料中解离, 这对于 开发新型基于石墨烯的纳米生物界面材料具有重要意

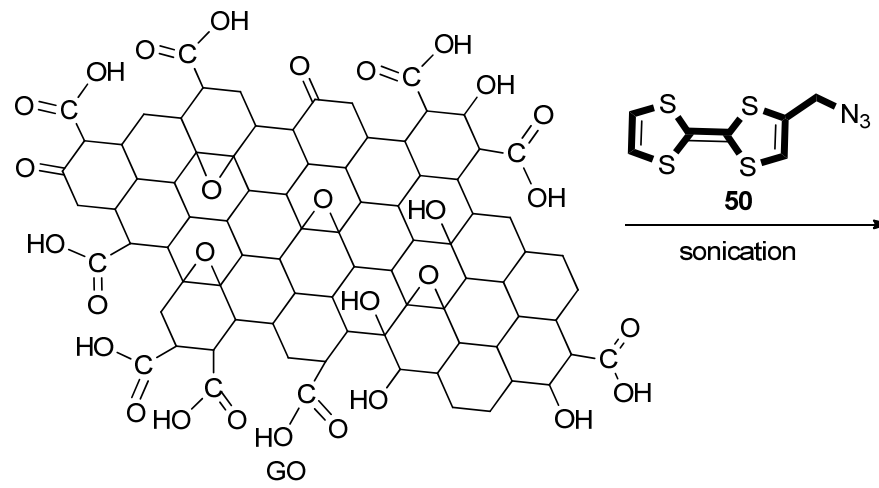
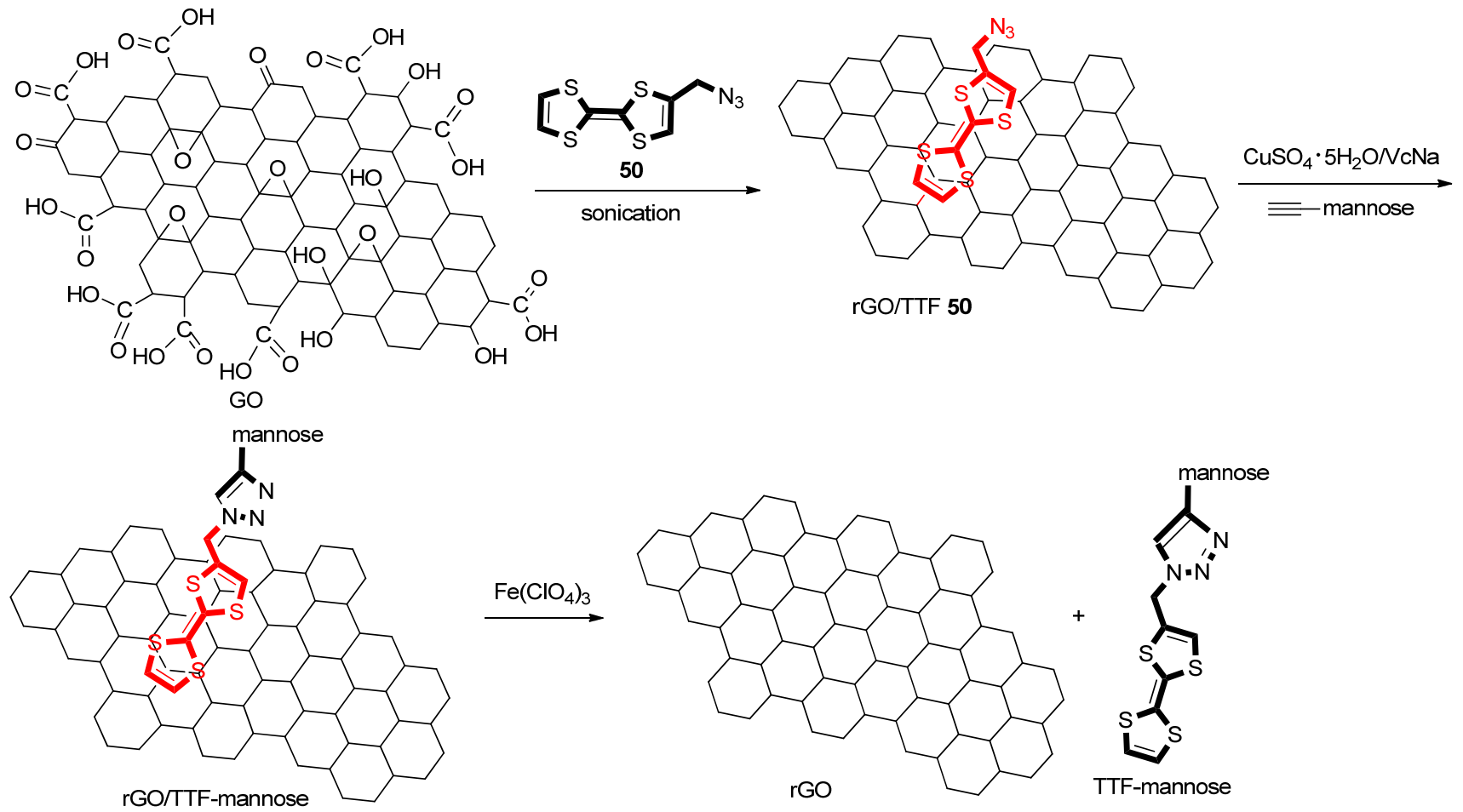

图式 9 基于糖-石墨烯材料的点击合成和氧化作用下糖的释放

Scheme 9 Formation of the clicked graphene/TTF-mannose nanosheets and the release of TTF-mannose from a graphene matrix by oxidation 
义。

2016 年, Emrick 等 ${ }^{[60]}$ 利用 $\mathrm{CuAAC}$ 点击反应, 将含 有端基炔的 TTF 行生物 51 与含有端基叠氮基的聚合物 反应得到含有电化学活性 TTF 单元(摩尔分数约为 $35 \%$ ) 的聚合物 52 (Eq. 11), 并研究了其与层状基面的 $\mathrm{MoS}_{2}$ 的作用行为. 结果表明含有 TTF 单元的聚合物的电化学 行为与单体 $\mathrm{TTF}$ 不同, 聚合物中 $\mathrm{TTF}$ 与 $\mathrm{MoS}_{2}$ 的作用促 进形成 TTF 自由基二聚体，进而扩大了聚合物表面键合 和静电作用行为, 对于开发利用层状 $\mathrm{MoS}_{2}$ 器件具有重 要意义.<smiles>[R]OC(=O)C(C)(CC(C)(C)C(C)(C)CC(=O)O)C(C)(C)C(=O)OCCN</smiles><smiles>C#CCOCC1=CSC(=C2SC=CS2)S1</smiles>
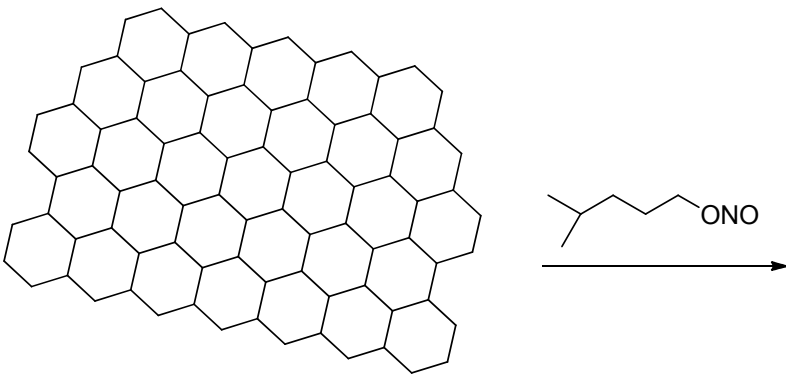

CNF (SWCNT, MWCNT, graphene)<smiles></smiles>

54
纳米科学和纳米技术的发展加快了富勒烯、碳纳米 管、石墨烯等碳纳米材料的开发与利用. 2015 年, Martín 等 ${ }^{61]}$ 利用自由基诱导碳纳米材料(CNF), 如单壁碳纳米 管(SWCNT)、多壁碳纳米管 $(\mathrm{MWCNT})$ 和石墨烯 $(\mathrm{G})$ 等发 生自由基加成反应得到含端基炔的纳米碳底物, 其再与 含端基叠氮基的 exTTF 衍生物 53 通过 CuAAC 点击反 应得到含有电化学活性单元的相应碳纳米功能(CNF)材 料 54 (Scheme 10). 电化学实验表明 exTTF 单元的呈现 双电子准可逆氧化还原过程. 光激发实验表明单壁碳纳

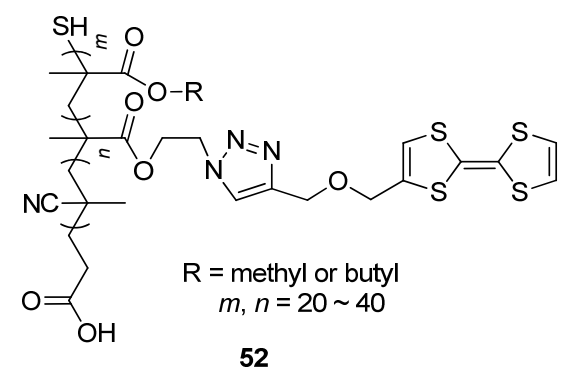

图式 10 含 exTTF 单元的碳纳米材料的点击合成

Scheme 10 Preparation of carbon nanoforms with exTTF units by CuAAC reaction 
米管(SWCNT) 54 可以形成电荷分离状态, 对于开发新 型光电材料具有重要意义.

\section{4 总结与展望}

点击化学是化学合成方法的重要里程碑, 其中 $\mathrm{CuAAC}$ 反应因具有原料易得、条件温和、产物立体选 择性好和产率较高等优势而获得广泛应用. 研究表明四 硫富瓦烯(TTF) 是优良的 $\pi$ 电子供体，其对 $\mathrm{CuAAC}$ 反应 的条件是适合的, 利用含端基炔的 TTF 化合物与含端基 叠氮基的底物反应或利用含端基叠氮基的 TTF 化合物 与含端基炔的底物反应可制备众多的含 1,2,3-三氮唑功 能基的 TTF 衍生物, 在识别传感、表面修饰、纳米材料、 太阳能电池材料和超分子功能器件等领域展现广泛的 应用前景. 可以期望随着研究的深入, CuAAC 点击反应 在 TTF 化学领域的研究更趋向于功能化和多学科化, 对 于拓展 TTF 的研究和应用具有重要意义.

\section{Referenes}

[1] Segura, J. L.; Martin, N. Angew. Chem., Int. Ed. 2001, 40, 1372.

[2] Canevet, D.; Sallé, M.; Zhang, G. X.; Zhang, D. Q.; Zhu, D. B. Chem. Commun. 2009, 45, 2245.

[3] Pop, F.; Avarvari, N. Chem. Commun. 2016, 52, 7906.

[4] Bendikov, M.; Wudl, F.; Perepichka, D. F. Chem. Rev. 2004, 104, 4891.

[5] Zhu, Y. L.; Cao, L.; Ma, K. R.; Tian, L. B.; Wang, X. L.; Su, Z. M. Chem. J. Chin. Univ. 2013, 34, 952.

[6] Bouzan, S.; Chen, G.; Mulla, K.; Dawe, L.N.; Zhao, Y. M. Org. Biomol. Chem. 2012, 10, 7673.

[7] Brunetti, F. G.; López, J. L.; Atienza, C.; Martín, N. J. Mater. Chem. 2012, 22, 4188

[8] Yamada, J.; Sugimoto, T. TTF Chemistry Fundamentals and Applications of Tetrathiafulvalene, Kodansha and Springer, Tokyo, 2004.

[9] Feng, M.; Gao, L.; Deng, Z. T.; Ji, W.; Guo, X. F.; Du, S. X.; Shi, D. X.; Zhang, D. Q.; Zhu, D. B.; Gao, H. J. J. Am. Chem. Soc. 2007, 129, 2204.

[10] Chen, T.; Liu, W. J.; Cong, Z. Q.; Yin, B. Z. Chin. J. Org. Chem. 2005, 25, 570 (in Chinese). (陈铁, 刘武军, 从志奇, 尹炳柱, 有机化学, 2005, 25, 570.)

[11] Zhu, Y. L.; Yang, Y. J.; Yin, Q. F.; Zhu, D. B. Chin. J. Org. Chem. 2005, 25, 1167 (in Chinese) (朱玉兰, 杨艳杰, 尹起范, 朱道本, 有机化学, 2005, 25, 1167.)

[12] Huo, J. P.; Wei, X. P.; Mo, G. Z.; Peng, P.; Zhong, M. L.; Chen, R. H.; Wang, Z. Y. Chin. J. Org. Chem. 2014, 34, 92 (in Chinese). (霍景沛, 韦新平, 莫广珍, 彭湃, 钟铭丽, 陈任宏, 汪朝阳, 有 机化学, 2014, 34, 92.)

[13] Rostovtsev, V. V.; Green, L. G.; Fokin, V. V.; Sharpless, K. B. Angew. Chem., Int. Ed. 2002, 41, 2596.

[14] Tornøe, C. W.; Christensen, C.; Meldal, M. J. Org. Chem. 2002, 67, 3057.

[15] Kolb, H. C.; Finn, M. G.; Sharpless, K. B. Angew. Chem., Int. Ed. 2001, 40, 2004.

[16] Moses, J. E.; Moorhouse, A. D. Chem. Soc. Rev. 2007, 36, 1249.

[17] Wu, P.; Fokin, V. V. Aldrichim. Acta 2007, 40, 7.

[18] Dondoni, A. Chem. Asian J. 2007, 2, 700.

[19] Meldal, M.; Tornøe, C. W. Chem. Rev. 2008, 108, 2952.

[20] Huisgen, R.; Szeimies, G.; Mobius, L. Chem. Ber. 1967, 100, 2494.

[21] Lutz, J. F. Angew. Chem., Int. Ed. 2007, 46, 1018.

[22] Angell, Y. L.; Burgess, K. Chem. Soc. Rev. 2007, 36, 1674.

[23] Sumerlin, B. S.; Vogt, A. P. Macromolecules 2010, 43, 1.
[24] Hahn, U.; Elhabiri, M.; Trabolsi, A.; Herschbach, H.; Leize,E.; Dorsselaer, A. V.; Albrecht-Gary, A. M.; Nierengarten, J. F. Angew. Chem., Int. Ed. 2005, 44, 5338 .

[25] Fournier, D.; Hoogenboom, R.; Schubert, U. S. Chem. Soc. Rev. 2007, 36, 1369.

[26] Biet, T.; Cauchy, T.; Avarvari, N. Chem. Eur. J. 2012, 18, 16097.

[27] Biet, T.; Avarvari, N. Org. Biomol. Chem. 2014, 12, 3167.

[28] Biet, T.; Avarvari, N. CrystEngComm 2014, 16, 6612.

[29] Salinas, Y.; Solano, M. V.; Sørensen, R. E.; Larsen, K. R.; Lycoops, J.; Jeppesen, J. O.; Martinez-Manez, R.; Sancenon, F.; Marcos, M. D.; Amorós, P.; Guillem. C. Chem.-Eur. J. 2014, $20,855$.

[30] Zhou, Y. C.; Zhang, D. Q.; Zhu, L. Y.; Shuai, Z. G.; Zhu, D. B. $J$ Org. Chem. 2006, 71, 2123.

[31] Li, X. H.; Zhang, G. X.; Ma, H. M.; Zhang, D. Q.; Li, J.; Zhu, D. B. J. Am. Chem. Soc. 2004, 126, 11543

[32] Mulla, K.; Dongare, P.; Thompson, D. W.; Zhao, Y. M. Org. Biomol. Chem. 2012, 10, 2542.

[33] Mulla, K.; Dongare, P.; Thompson, D. W.; Zhao, Y. M. Org. Lett. 2013, 17, 4532 .

[34] Jelinek, R.; Kolusheva, S. Chem. Rev. 2004, 104, 5987.

[35] Edwards, N. Y.; Sager, T. W.; McDevitt, J. T.; Anslyn, E. V. J. Am. Chem. Soc. 2007, 129, 13575.

[36] Tan, W.; Zhang, D.; Wang, Z.; Liu, C.; Zhu, D. J. Mater. Chem. 2007, 17, 1964.

[37] Shoji, E.; Freund, M. S. J. Am. Chem. Soc. 2002, 124, 12486.

[38] Mulla, K.; Zhao, Y. M. Tetrahedron Lett. 2014, 55, 382.

[39] Shao, M.; Zhao, Y. M. Tetrahedron Lett. 2010, 51, 2508

[40] Qvortrup, K.; Petersen, M. A.; Hassenkam, T.; Nielsen, M. B. Tetrahedron Lett. 2009, 50, 5613.

[41] Zhao, B. T.; Liu, L. W.; Li, X. C.; Qu, G. R. Chin. J. Chem. 2012, 30,254 .

[42] Zhao, B. T.; Liu, L. W.; Li, X. C.; Qu, G. R.; Belhadj, E.; Le Derf, F.; Sallé, M. Tetrahedron Lett. 2013, 54, 23

[43] Zhao, B. T.; Cao, S. N.; Guo, H. M.; Qu, G. R. Synth. Met. 2013, $174,14$.

[44] Liu, L. W.; Guo, W. B.; Li, X. C.; Qv, G. R.; Zhao, B. T. Chin. J. Org. Chem. 2010, 30, 1960 (in Chinese).

(刘连委，郭文博，李晓川，渠桂荣，赵邦屯，有机化学，2010, 30, 1960.)

[45] Zhao, B. T.; Zhu, X. M.; Chen, X. H.; Yan, Z. N. Chin. Chem. Lett. 2013, 24, 573

[46] Zhao, B. T.; Peng, Q. M.; Zhu, X. M.; Yan, Z. N.; Zhu, W. M. J. Org. Chem. 2015, 80, 1052.

[47] Xue, M.; Yang, Y.; Chi, X. D.; Yan, X. Z.; Huang, F. H. Chem. Rev. 2015, 115,7398

[48] Spruell, J. M.; Paxton,W. F.; Olsen, J. C.; Benítez, D.; Tkatchouk, E.; Stern, C. L.; Trabolsi,A.; Friedman, D. C.; Goddard, W. A.; Stoddart, J. F. J. Am. Chem. Soc. 2009, 131, 11571.

[49] Fahrenbach, A. C.; Hartlieb, K. J.; Sue, C. H.; Bruns, C. J.; Barin, G.; Basu, S.; Olson, M. A.; Botros, Y. Y.; Bagabas, A.; Khdaryad, N. H.; Stoddart, J. F. Chem. Commun. 2012, 48, 9141.

[50] Andersen,S. S.; Share, A. I.; Poulsen, B. C.; Kørner, M.; Duedal, T.; Benson, C. R.; Hansen, S. W.; Jeppesen, J. O.; Flood, A. H. J. Am. Chem. Soc. 2014, 136, 6373.

[51] Baggerman, J.; Haraszkiewicz, N.; Wiering, P. G.; Fioravanti, G.; Marcaccio, M.; Paolucci, F.; Kay, E. R.; Leigh, D. A.; Brouwer, A. M. Chem.-Eur. J. 2013, 19, 5566.

[52] Avellini, T.; Li, H.; Coskun, A.; Barin, G.; Trabolsi, A.; Basuray, A. N.; Dey, S. K.; Credi, A.; Silvi, S.; Stoddart, J. F.; Venturi, M. Angew. Chem., Int. Ed. 2012, 51, 1611.

[53] Aprahamian, I.; Dichtel, W. R.; Ikeda, T.; Heath, J. R.; Stoddart, J. F. Org. Lett. 2007, 7, 1287.

[54] Aprahamian, I.; Yasuda, T.; Ikeda, T.; Saha, S.; Dichtel, W. R.; Isoda, K.; Kato, T.; Stoddart, J. F. Angew. Chem., Int. Ed. 2007, 46, 4675 .

[55] Aprahamian, I.; Olsen, J. C.; Trabolsi, A.; Stoddart, J. F. Chem.-Eur. J. 2008, 14, 3889 .

[56] Zhao, Y. L.; Dichtel, W. R.; Trabolsi, A.; Saha, S.; Aprahamian, I.; 
Stoddart, J. F. J. Am. Chem. Soc. 2008, 130, 11294.

[57] Zhang, Q.; Tu, Y, Q.; Tian, H.; Zhao, Y. L.; Stoddart, J. F.; Ågren, H. J. Phys. Chem. B 2010, 114, 6561.

[58] Barin, G.; Coskun, A.; Friedman, D. C.; Mark, A.; Olson, D.; Michael, T.; Colvin, R.; Sanjeev, K.; Dey, M. A.; Altan, B.; Michael, R.; Wasielewski, G.; Stoddart, J. F. Chem.-Eur. J. 2011, 17, 213.

[59] Kaminska, I.; Barras, A.; Coffinier, Y.; Lisowski, W.; Roy, S.; Niedziolka-Jonsson, J.; Woisel, P.; Lyskawa, J.; Opallo, M.; Siri- wardena, A.; Boukherroub, R.; Szunerits, S. ACS Appl. Mater. Interf. 2012, 4, 5386.

[60] Selhorst, R. C.; Puodziukynaite, E.; Dewey, J. A.; Wang,P. J.; Barnes, M. D.; Ramasubramaniam, A.; Emrick, T. Chem. Sci. 2016, 7, 4698.

[61] Mateos-Gil, J.; Rodríguez-Pérez, L.; Guldi, D. M.; Herranz, M. A.; Martín, N. Nanoscale 2015, 7, 1193.

(Cheng, F.) 